\title{
States and unknown input estimation via non-linear sliding mode high-gain observers for a glucose-insulin system
}

\begin{abstract}
:
A meal-estimation algorithm is developed based on an extended mathematical model of the glucose-insulin system. The proposed model describes the dynamics of glucose levels in blood and in subcutaneous layer, as well as the meal intake which is considered an unknown input of the system. This model seeks to represent in a more realistic manner, the pancreas malfunction in patients with Type 1 Diabetes Mellitus. Based on model, a non-linear high gain observer (NHGO) with a sliding mode is designed in order to estimate the unmeasured states and the external disturbances of the system. This scheme is useful to maintain frequent monitoring of glucose levels and any changes in its behaviour. The unknown input or disturbance is estimated through the sliding mode based only the estimation error. Data from a real patient is used to evaluate the effectiveness of the proposed estimation scheme.
\end{abstract}

